\title{
A Dual-Component View of Propositional Grasping
}

\author{
John Dilworth • Dylan Sabo
}

Received: 9 July 2012/Accepted: 5 August 2013/Published online: 6 September 2013

(C) Springer Science+Business Media Dordrecht 2013

\begin{abstract}
On a traditional or default view of the grasping or understanding of a singular proposition by an individual, it is assumed to be a unitary or holistic activity. However, naturalistic views of cognition plausibly could analyze propositional thinking in terms of more than one distinctive functional stage of cognitive processing, suggesting at least the potential legitimacy of a non-unitary analysis of propositional grasping. We outline a novel dual-component view of this kind, and show that it is well supported by current cognitive science research.
\end{abstract}

Issues concerning the structure and constitution of singular propositions (the only kind of proposition discussed here) have usually been addressed on broadly logical or metaphysical grounds. For example, the modal view of a proposition as a set of possible worlds explains a proposition as being the set of the worlds in which it is true. A traditional structured view of a proposition would regard it as an ordered pair of the particular object it is about, plus either a property extension-set, or the property itself that the proposition attributes to the object. And so on.

Nevertheless, it has also been generally agreed that propositions must be such that they are capable of being grasped or understood by humans. For if propositions are to be truth-bearers, to explain the contents of our beliefs, and so on, presumably there must potentially be some adequate account of how we can stand in appropriate relations to them-whether of epistemic or other kinds-that would permit propositions to have the explanatory roles for us that they do in fact have. But as a consequence, these relational constraints would also require appropriate constraints

\footnotetext{
J. Dilworth $(\bowtie)$

Department of Philosophy, Western Michigan University, Kalamazoo, MI 49008, USA

e-mail: dilworth@wmich.edu

D. Sabo

Department of Philosophy, Occidental College, Los Angeles, CA 90041, USA

e-mail: sabo@oxy.edu
} 
on possible propositional structures, in order that they could serve our cognitive and epistemic purposes. For example, it is generally assumed that propositions at least must have a subject-predicate structure, in order to explain the fact that our thoughts generally seem to take this form.

Presumably some form of this 'cognitive constraint' thesis for propositional structure would also be generally agreed on. But there might also be some respect in which more than one kind of propositional structure potentially would be consistent with adequate propositional grasping. We shall argue that this is the case for a novel dual-component versus a traditional single-component conception of propositional structure. The issue may be introduced as follows.

The traditional assumption has been, by default, that cognitive grasping of a proposition is of a holistic, all-or-nothing kind-either a cognizer grasps a complete proposition, or grasps nothing that is specifically propositional. So on this traditional view, there is nothing in the structure of propositional grasping itself that could provide any clues as to the nature of propositional structure, beyond an assumption that the structure of a proposition is as unified as the cognitive act that grasps it. For convenience this traditional view will be called grasping holism.

However, if we turn instead to empirical issues regarding the cognitive processing of worldly information, such as a perceptual process that results in the perceiver seeing that an object $\mathrm{x}$ is an $\mathrm{F}$, clearly it involves a sequential causal process that potentially could be broken down into stages, each of which might have a distinctive functional role in the overall informational process. Consequently, it is at least empirically possible that there could be more than one distinctive stage in the grasping of a perceptually derived proposition as well-so that the propositional grasping has an articulated structure that involves more than one of these intermediate stages of cognitive processing of the relevant information. In Sects. 3, 4 and 5 we shall present some empirical evidence that tends to support such an articulated view.

\section{The Dual Structure of a Grasped Proposition}

Our proposal will be that cognitive constraints involved in cases of perceptuallyderived singular propositions support a two-component or dual structure for such a proposition. Thus on our view, grasped propositions are grasped as dual-component propositions, or D-propositions for short.

As a rough initial overview-to be refined below-consider the proposition that an object $\mathrm{x}$ is an $\mathrm{F}$, where $\mathrm{F}$ is some qualitative or sortal property such as that of being red, of being a man, and so on. For example, the proposition that $\mathrm{x}$ is a man claims, of some actual worldly object $x$, that it has the property of being a man. Our proposal will be that the relevant propositional claim about that actual object $\mathrm{x}$ can, with respect to cognitive grasping of it, be broken down into two distinctive grasped components. The first content-based component involves a grasping of something as being a man. The second, actuality-based component involves the grasping of a relevant worldly or actual fact-namely, the fact of whether the particular actual object $\mathrm{x}$ is, or is not, a man. 
Consequently, a complete grasping of a full, truth-evaluable singular proposition is a grasping of whether or not the first component corresponds with the second component-which correspondence holds just in case each has the same predicative content. Nevertheless, in our view only the first, content-based propositional component is directly or immediately grasped as a whole. We shall now describe how the full grasping of the proposition, along with its correspondence-based truthconditions, would involve at least two stages of cognitive inference from items that are immediately cognitively available in paradigm perceptual cases.

The first stage of cognitive inference is required because the second, actual-fact component of a proposition is not itself immediately available to cognition in perceptual situations. All that is immediately available is-we shall argue-a causally-based referential link or pointer to the relevant worldly object $\mathrm{x}$ that the proposition is about. Or, in more familiar philosophical terms, all that is available is a pure perceptual demonstrative, having a nonconceptual content (see Sect. 3 for empirical support of this view). Consequently, the immediate cognitive grasping of such a referential link must be supplemented with an inference to there being some salient worldly fact-involving that object $\mathrm{x}$-which would be relevant to establishing whether or not the full proposition that $\mathrm{x}$ is an $\mathrm{F}$ is true or false. Thus the second propositional component is only indirectly grasped, via an inferential construction of this kind that builds on an immediate grasp of a referential pointer to the worldly object $\mathrm{x}$.

The second required inferential construction takes the two propositional components already arrived at-namely, the immediate content-based component, and the inferentially-constructed worldly-fact-based component-and construes them in turn as a complete proposition whose truth-value depends on whether the first component corresponds with the second component.

As a result, our dual-component proposal as a whole provides a fairly intuitive, though unconventional, version of a correspondence-based account of propositional truth as depending on correspondence with a worldly fact. On our account, grasping a proposition involves grasping each of its two components, along with grasping that the truth-condition for the thus-grasped proposition is that the first, contentbased component should correspond with the second, actual-fact component.

Nevertheless, this remains a non-traditional and non-unitary conception of a proposition and of its correspondence-based truth. Our D-propositional claim is not the traditional claim that a unitary proposition is true when it corresponds with an actual fact. Instead our claim is that a D-proposition is true when its first, contentbased component corresponds with the actual fact specified by its second component. Or, more precisely, our claim is that what it is for a cognizer $\mathrm{C}$ to grasp a D-proposition and its truth-condition is for $\mathrm{C}$ to grasp its first component, and to grasp that whether or not the complete D-proposition is true or not depends on whether that first grasped component corresponds with the second grasped component.

The non-unitary nature of D-propositions can be further emphasized as follows. Neither of the two components of a D-proposition is fully propositional on its own-so that each is logically unsaturated, in that neither component determines a unique proposition by itself. The content component is unsaturated, in that by itself 
it does not determine any particular actual object that a full proposition would be about. And the actual-fact component is unsaturated, in that by itself it does not determine a truth-value for the relevant full proposition concerning object $\mathrm{x}$.

However, we should emphasize that these initial formulations of D-propositional structure provide no more than a rough initial overview, which would be subject to various clarifications and emendations in future, more detailed work on these topics.

\section{A Naturalistic Account of Propositional Grasping}

Before proceeding further, a standard objection to grasping-based accounts of propositional cognition should be mentioned. The objection is that since propositions are generally assumed to be abstract entities, they could have no place in a naturalistic explanatory account of any kind of concrete cognitive processing. Thus the objection is that the concept of cognitive grasping of a proposition must at best be a picturesque metaphor, rather than a specification of a legitimate factor in a literal account of some naturalistically-specifiable cognitive activities. For example, Fodor argues that he can make no sense of the idea of grasping an abstract proposition, so instead he substitutes concrete cognitive relations to mental representations that consist of token mental sentences in a language of thought (LOT) — which sentences have relevant propositions as their contents, but which propositions play no causal role in his naturalistic theory (Fodor 1975, 1978).

We accept this objection-namely, that a strictly cognitive account of propositional grasping or understanding must explain it in purely naturalistic terms, and hence that such an account must provide an adequate substitute rather than a strict equivalent for the concept of grasping an abstract proposition. Here is an overview of how we shall proceed, with the relevant objection being duly noted (thus our future references to propositional grasping will address substitute rather than abstract versions).

Our account is structured around a basic distinction between two different kinds of categorization or classification by a cognizer-namely, stimulus versus object classification. The basic idea is that an experienced cognizer would have abilities to classify some structured incoming perceptual stimuli in conceptual terms-hence producing a conceptualized cognitive model (CCM) that is a conceptualized or categorized form of structured perceptual data derived from sensory inputs.

Now at this early stage of cognitive processing, a relevant CCM-such as a conceptualized model of something as being an $\mathrm{F}$-would not represent, in a truthevaluable way, any actual worldly item as being an F. Instead, it merely would categorize some incoming perceptual data as being F-related data. Our view is that such an early-stage CCM is capable of providing a concrete form of the first, content-based component in our proposed D-propositional structure. A cognitive system that is currently processing such an F-related CCM is thereby in a cognitive state in which it grasps that something or other is classifiable in F-related termshence providing the first grasped component in our proposed account of grasping a D-proposition. (See the following sections for empirical support for this hypothesis). 
We turn now to the other kind of categorization-namely object categorization, or more specifically, categorization of a particular worldly object $\mathrm{x}$ as being an F. In our view this is a fully representational kind of categorization that has a singular, truth-evaluable propositional content-which categorization is the final result of further cognitive processing having been applied to the initial CCM stage as just discussed. Thus our account-unlike more standard naturalistic accounts of mental representation-emphasizes the integral role of intermediate perceptual processing, plus of the two kinds of inferential processing discussed in the previous section, in arriving at a full mental representation whose propositional content is graspable as the proposition that a particular worldly object $\mathrm{x}$ is an $\mathrm{F}$.

But what is wrong-if anything-with more standard holist views of propositional grasping, which ignore such intermediate or inferential stages of cognitive processing? In this paper we aim only to show, in a preliminary way, that there are significant empirical results that support a dual, D-propositional view, but which results would remain unaccounted for on more standard holist propositional grasping views.

\section{Grasping an Object Via Nonconceptual Demonstrative Reference}

This section provides some empirical evidence for our claim in Sect. 1 that the second, actual-fact component of a proposition-concerning the putative Fness of an object $\mathrm{x}$-is not immediately graspable in its entirety in perceptual situations. Instead, all that is immediately cognitively graspable is one factor in that second component-namely, a causally-based pure referential demonstrative that points to the relevant worldly object $\mathrm{x}$ that the proposition is about. ${ }^{1}$

A major source of evidence for this claim it provided by the work of Zenon Pylyshyn and associates on the phenomenon of multiple object tracking by perceivers, which is addressed by Pylyshyn's FINST visual indexing theory (Pylyshyn and Storm 1988; Pylyshyn 2000, 2001, 2007, 2009; Raftopoulos and Muller 2006; Fodor 2009).

The FINST theory is based on strong evidence that singular perception is causally grounded in FINSTs (visual indexes or pointers) that track particular worldly objects-but it is only the objects, and not their properties as well, that are tracked. According to Pylyshyn in his latest overview of his theory (2009), the existence of this foundational kind of purely object-based, nonconceptual referential causal tracking is theoretically compelling for various reasons, ${ }^{2}$ including that it can provide a purely naturalistic basis for theories of mental representation, without any question-begging assumptions concerning the nature of concepts, the mechanisms

\footnotetext{
${ }^{1}$ For evidence that such a demonstrative could provide a legitimate kind of cognitive acquaintance or grasping of an object, see Dickie (2010).

2 Such as the limited capacity of the mind to process information, the reality that representations must be constructed incrementally, the need to solve the binding problem of how properties are assigned to a particular object, and the need to tag individuals to mark them during visual processing (2009, p. 9-11).
} 
of linguistic reference, and so on-a point whose importance is also enthusiastically endorsed by Fodor in his commentary on Pylyshyn's views (Fodor 2009, p. xiv). ${ }^{3}$

In addition, Raftopoulos's comprehensive book (2009) provides further convincing arguments to the effect that the reference of perceptual demonstratives is fixed in a causal, purely nondescriptive way through the nonconceptual content of perception. On his account, "The causal definition of nonconceptual content is independent of any considerations about the conceptual arsenal that the perceiver might have or might exercise while she perceives" (p. 153).

However, as Pylyshyn acknowledges, though a FINST-style account explains how a mental object file could be opened for a particular worldly object that was tracked, it provides no account of how conceptual information concerning the object could subsequently be associated with the file (Pylyshyn 2009, p. 5-6). Our hypothesis-as briefly outlined in Sect. 1-is that such conceptual information is inferred from prior, independent processes of perceptual categorization, derived from mental processing associated with the first, content-based component of a relevant proposition. In addition, see Sect. 6 for a summary of the role of Pylyshynstyle perceptual demonstratives in supporting our account of propositional structure.

\section{The Independence of Perceptual Categorization from Object Recognition}

Our D-propositional thesis that grasping a singular proposition involves grasping two independent propositional components stands or falls with the availability of evidence that there is an early stage of non-truth-evaluable cognitive categorization that is independent of, and temporally prior to, the formation of a complete truthevaluable mental representation whose propositional content is the proposition that a particular worldly object $\mathrm{x}$ is $\mathrm{F}$ (or: an F).

In particular, in perceptual cases we seek evidence that cases of perceptual categorization are not already full-blown cases of worldly object recognition of an object $\mathrm{x}$ as being of some kind $\mathrm{F}$-for if they were such recognitional cases, they would already have a complete graspable propositional content, and so fail to provide empirical evidence that there could be two independent graspable propositional components. Thus we seek evidence supporting a prior independence thesis for one component of a proposition-which component should not itself be a complete proposition.

Our prior independence thesis is related to Pylyshyn's nonconceptual FINST visual index thesis in the following way. We can agree with Pylyshyn that singular object tracking is a well-confirmed feature of early perceptual processing, so that the formation of a singular, FINST-based object file for a particular worldly object $\mathrm{x}$ may take place at approximately the same time as initial processes of perceptual categorization. Nevertheless, our claim is that any descriptive information associated with that object file does not become associated with it until after initial

\footnotetext{
3 "In short, we need to explain how there can be nonconceptual reference. I think Pylyshyn's recent work shows how one might proceed in the direction of such an account... If that's so, then it's the best idea about the semantics of mental representation than [sic] anybody has ever had."
} 
processes of perceptual categorization have occurred. Our overall thesis requires this temporal offset, for we assume that once a process of association of descriptive information with a FINST-based object file has been completed, there would already exist a complete, propositionally based mental representation of object $\mathrm{x}$.

Here is some empirical evidence for our prior independence thesis. Vanrullen and Thorpe (2001) provide some cognitive neuroscience evidence both for the temporal priority part of our thesis, and for the independence part. On the temporal priority issue, they summarize their results as follows: “...we show that visual categorization of a natural scene involves different mechanisms with different time courses: a perceptual, task-independent mechanism, followed by a task-related, categoryindependent process. Although average ERP (event-related potential) responses reflect the visual category of the stimulus shortly after visual processing has begun (e.g. 75-80 ms), this difference is not correlated with the subject's behavior until $150 \mathrm{~ms}$ poststimulus." (p. 454).

We interpret this result as follows. In naturalistic theories of mental representation, such as Fodor's Representational Theory of Mind (1975, 1987, 2008), the formation of a complete mental representation having a propositional content is closely associated with the causal role of that mental representation in generating an appropriate behavioral response. So the fact that such behavioral responses occur at a significantly later time than the formation of an initial perceptual categorization is evidence that the perceptual categorization belongs to an earlier, pre-propositional phase of processing.

The same authors (VanRullen and Thorpe 2001) also summarize recent evidence (e.g., Romo and Salinas 1999; Schall and Thompson 1999; Shadlen and Newsome 1996) relevant to the independence issue, as follows: "Current theories of visual processing suggest a distinction between two different mechanisms: a perceptual process extracting information about different properties of the visual input, followed by a higher-level decision process evaluating the relevance of this visual information, in terms of the goals and expectations of the subject, in order to prepare and generate the appropriate behavioral response." (p. 454). Their summary in terms of levels of processing provides another way in which to distinguish the early perceptual categorization processes from the later, more cognitive and fully propositional processes.

Other cognitive scientists supporting related prior independence theses include Liu et al. (2002) ${ }^{4}$; Curran et al. (2002) $)^{5}$; and important earlier work by Warrington and associates on evidence of separations of perceptual categorization from later semantic categorization (e.g., Warrington and Taylor 1978; Warrington 1982) that builds on work of de Renzi et al. (1969), who, as Warrington points out, “...reported the first quantitative evidence of a double dissociation between what I

\footnotetext{
${ }^{4}$ Part of their summary: "These data suggest that face processing proceeds through two stages: an initial stage of face categorization, and a later stage at which the identity of the individual face is extracted." (p. 910).

5 Part of their conclusion: "The clearest general result to emerge from the present experiments is a temporal sequence of events involving a transition between early sensitivity to category membership (in/ out differences) and later sensitivity to differential experience with particular exemplars (old/new differences). This temporal sequence suggests that the information necessary for categorization may become available earlier than that necessary for recognition memory" (p. 14).
} 
have termed perceptual categorization and semantic categorization." (Warrington 1982, p. 30).

But probably the most philosophically sophisticated account of the timing differences of earlier perceptual categorization versus later, propositionally related cognition is to be found in the work of Raftopoulos, much of which is presented in his recent book (2009), as mentioned in the previous section. His complex investigations resist any brief summary, but a subsequent article (Raftopoulos 2011) is explicit on the time gap between initial conceptualization and subsequent belief formation, as follows: "Conceptual information about a tiger affects visual processing and after some hypothesis testing the animal is recognized as a tiger through the synergy of visual circuits and WM (working memory). At this point the explicit belief "O is F" is formed. This occurs after $300 \mathrm{~ms}$, when the viewer consolidates the object in WM and identifies it with enough confidence to report it, which means that beliefs are formed at the final phases of late vision. However, semantic modulation of visual processing and the process of conceptualization that eventually leads to object recognition starts at about 130-200 ms. There is, thus, a time gap, between the onset of conceptualization and the recognition of an object, which is a prerequisite for the formation of an explicit recognitional belief." (p. 7).

\section{Other Kinds of Evidence}

This section provides a brief summary of more miscellaneous kinds of evidence that perceptual categorization is only one incomplete and separable stage in the formation of singular, fully propositional mental representations. Though the focus here is not on evidence of temporal priority, such priority is nevertheless implied or presupposed in many cases, in that generally there would be no point in a cognitive system processing incomplete or proto-propositional information unless the information could subsequently play a causal role in the formation of fully propositional information.

To begin, there is the whole weight of tradition in philosophy and cognitive science that separates issues concerning perceptual processing from issues concerning cognition more generally, including object recognition-which is one integral factor in the cognitive structure of propositional knowledge about the world. For example, as Palmieri and Gauthier (2004), p. 291-292 point out, in the psychological literature there is an almost complete separation between studies addressing perceptual categorization issues, and studies addressing object recognition issues.

This division between perceptual categorization versus cognitive thought issues is reinforced by the widely accepted view that perception, unlike thought, is modular or informationally encapsulated (Fodor 1983; Pylyshyn 1999; Raftopoulos 2009). If the encapsulation thesis is correct, it provides further reasons to distinguish early, encapsulated perceptual categorization from later cognitive, non-encapsulated and fully propositional kinds of categorization.

Another important characteristic of perceptual processes is that they are closely related to processes of mental imagery (Strawson 1974; Kosslyn 1994; Kosslyn et al. 2006; Pylyshyn 2003, 2007). A useful test case for our purposes is Nanay's 
(2010) demonstration that cases of amodal perceptual completion-such as when a partly occluded shape is mentally represented as a single whole object [see the occluded horse shape or shapes in the diagram (ibid., p. 244)]—cannot adequately be explained as involving a fully propositional belief about the hidden object or objects, but instead such cases are best explained as involving mental imagery that is not fully propositional or belief-like.
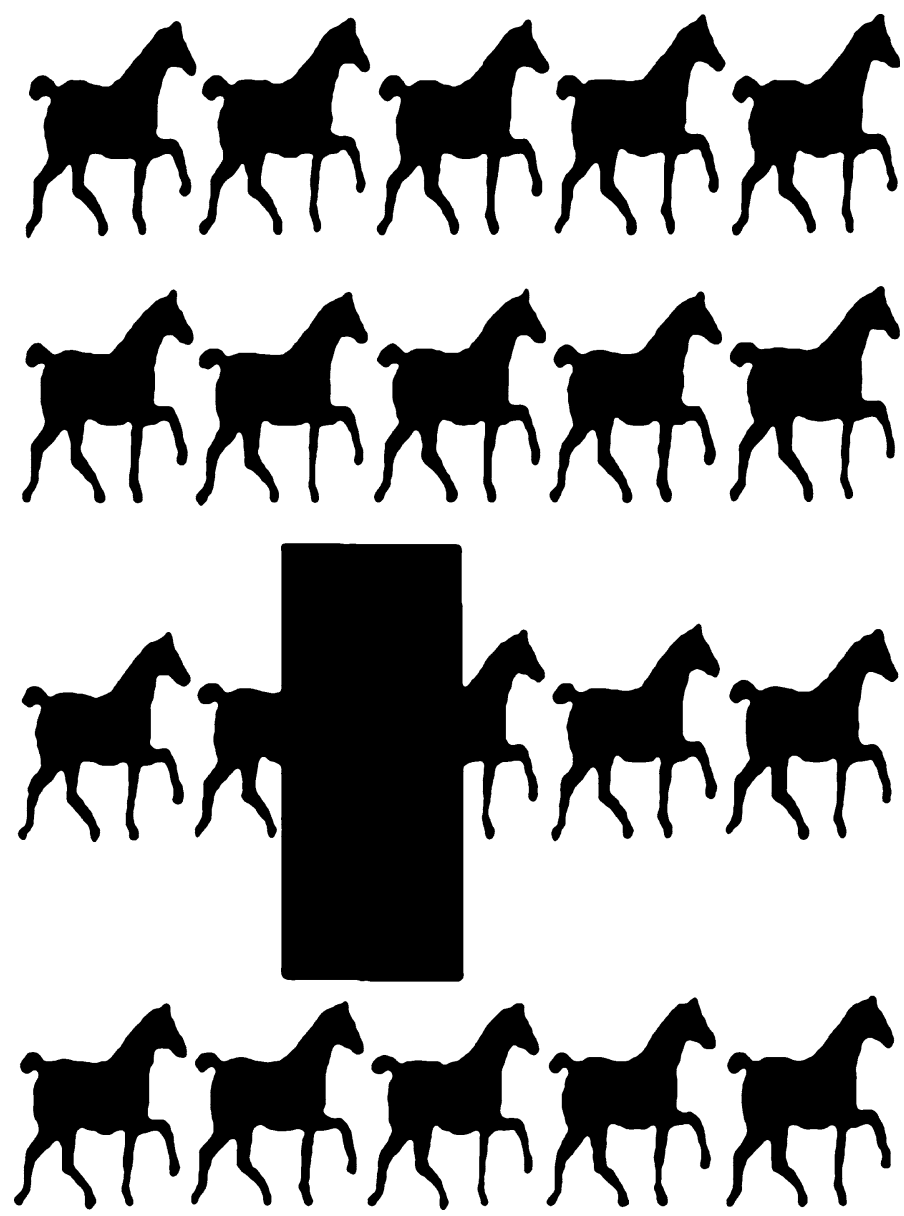

One reason for Nanay's conclusion may be paraphrased as follows. What needs to be explained is why almost all test subjects see the occluded horse shape or shapes as being a case of a single, long occluded horse. ${ }^{6}$

\footnotetext{
${ }^{6}$ Perry discusses a similar, dog-based example in his book (2001, 2nd ed. 2012: section 4.5, "Stretch the dog" and section 5.6, "Stretching cognitive content") in the broader philosophical setting of a distinction between indexical and referential content. Indeed, Pylyshyn (2009), p. 8 acknowledges the close relation between Perry's work on the ineliminability of indexicals and his own more empirical, FINST-based conclusions on demonstratives. Perry also assumes, as do Nanay's test subjects, that it is natural to see a single stretched animal in such partial occlusion cases.
} 
This is unlikely to be an inferentially supported belief, because-as the rest of the array of horse shapes attests - the only plausible belief is that two distinct horses are being occluded, given the sizes and placement of the other horse shapes in the array. However, it is known that mental imagery tends-independently of higher-level beliefs - to complete partially occluded shapes or objects in the simplest possible way, which here would involve imagery of a single, partially occluded horse. Hence the imagery-based account provides the most plausible hypothesis as to why subjects report seeing a single, stretched horse in the relevant case. ${ }^{7}$ Thus in Nanay's view we must distinguish between the quasi-perceptual and nonpropositional process of e.g. having a mental image of an apple, versus a fully propositional case of imagining that there is an apple in the kitchen (ibid., p. 249). Also, since in Nanay's view all visual perception involves mental imagery of the occluded aspects of seen objects, perceptual processing in general must involve integral, non-propositional kinds of categorization of objects.

More generally, our D-proposition theory potentially offers a way to mediate between those who hold that mental imagery is non-propositional and nondescriptive-such as Kosslyn and Nanay-and those such as Pylyshyn who argue that the empirical data just as well supports a purely descriptive account of the mental processing of imagery. Our mediation could concede to Pylyshyn that imagery does have descriptive content, but that this is a non-propositional kind of descriptive content that in perceptual cases would be derived from early perceptual categorization, prior to fully propositional object recognition. Consequently, Kosslyn, Nanay et al. could also be correct in holding that imagery content is distinctive, in that it is not fully propositional.

\section{The Predicative Division of Labor}

We have now assembled enough evidence in the previous three sections to make it plausible that propositional thinking could involve, among other things, the grasping of an initial propositional component that is not, by itself, fully propositional or truth-evaluable. This section will briefly motivate the rest of the propositional structure suggested in the opening sections of this paper.

To begin, if Pylyshyn and his supporters are correct-as discussed in Sect. 3that perceptually based reference is achieved exclusively through a nonconceptual kind of demonstrative worldly reference to particular objects, then the following structural problem arises. On the traditional view, a singular proposition involves both reference to a particular worldly object $\mathrm{x}$, and predicative information concerning some property or properties of that same object x. However, assuming that Pylyshyn et al. are correct about the causal basis of worldly reference, strictly speaking no direct predicative information about a particular worldly object $\mathrm{x}$ could be derived through perception. For example, on Pylyshyn's FINST-based view,

\footnotetext{
7 Further confirmation of Nanay's view that amodal completion is non-propositional is provided by Campbell (2006), who cites much evidence showing that "Amodal completion is one of the early processes in vision, and does not seem to depend on the top-down influence of the subject's conceptual thought" (ibid, p. 229).
} 
strictly speaking we never directly see properties of objects such as redness or manhood-all that we see is some visually identified worldly patch or putative object $\mathrm{x}$, about which we come to believe that it-i.e., object $\mathrm{x}$ - is red, or a man.

Our suggestion is that this highly paradoxical state of affairs-of the direct predicative unavailability of worldly properties of particular objects-must be resolved by appeal to some more indirect way in which we could come to grasp worldly properties or predicates as belonging to a particular object in a truthevaluable manner. The 'predicative division of labor' suggested in the title of this section points to our way of resolving the situation. Instead of directly perceiving a worldly property $\mathrm{F}$ of a particular object $\mathrm{x}$, we hypothesize that the cognitive process is broken down into two stages, so that there is a division of labor between the two stages in specification of the relevant predicative information, as follows (see the opening sections of the paper for an initial outline of the relevant structures).

In the first processing stage, some initial perceptual data is categorized as being F-related data-but at this early stage, this predicative categorization is not yet associated with any actual worldly object x. This initial, perceptual categorization stage is associated with the initial propositional component whose content could be described as that of 'something being F' - which does not have a full truth-value, because by itself it does not represent that there is any worldly object that has the relevant property. Consequently, it is not equivalent to a singular proposition that a particular worldly object $\mathrm{x}$ is an $\mathrm{F}$, nor to an existentially quantified proposition that some worldly object or other is an F.

Then a second processing stage involves an inference to the effect that the relevant causally-based, direct referential pointer to a worldly object $\mathrm{x}$ (as discussed in Sect. 3) is associated with a salient worldly fact concerning object $\mathrm{x}$-namely, whether or not it is actually an F. The propositional component associated with the result of this inference involves an F-related worldly fact-but, as with the first propositional component, this component by itself is not fully propositional either, in that a full proposition requires a correspondence condition that an actual fact by itself could not provide.

Consequently, as discussed previously, the grasping of the full proposition requires two inferential stages - first an inference to there being an F-related actual fact concerning object $\mathrm{x}$, and second, an inference to there being a full proposition, whose truth-condition depends on whether or not the first grasped propositional component corresponds with the second component. Or, to put the matter in more colloquial, beliefbased terms, a perceptually derived propositional belief concerning an object x involves believing that how something perceptually seems to be-namely, something perceptually seeming to be an $\mathrm{F}$-corresponds with how the relevant worldly object $\mathrm{x}$ actually is with respect to its being or not being F. It will be work for future papers to investigate further the grasping of D-propositional structures as proposed and supported here.

\section{References}

Campbell, J. (2006). Does visual reference depend on sortal classification? Reply to Clark. Philosophical Studies, 127, 221-237. 
Curran, T., Tanaka, J., \& Weiskopf, D. (2002). An electrophysiological comparison of visual categorization and recognition memory. Cognitive, Affective, \& Behavioral Neuroscience, 2, 1-18.

De Renzi, E., Scotti, G., \& Spinnler, H. (1969). Perceptual and associative disorders of visual recognition: relationship to the site of the cerebral lesion. Neurology, 19, 634-642.

Dickie, I. (2010). We are acquainted with ordinary things. In R. Jeshion (Ed.), New essays on singular thought (pp. 213-245). Oxford: Oxford University Press.

Fodor, J. (1975). The language of thought. Cambridge, MA: Harvard University Press.

Fodor, J. (1978). Propositional attitudes. Repr. in his Representations (pp. 177-203). Cambridge, MA: MIT Press.

Fodor, J. (1983). The modularity of mind. Cambridge, MA: MIT Press.

Fodor, J. (1987). Psychosemantics. Cambridge, MA: MIT Press.

Fodor, J. (2008). Lot 2. Oxford: Oxford University Press.

Fodor, J. (2009). Introduction: So what's so good about Pylyshyn? In D. Dedrick \& L. Trick (Eds.), Computation, cognition, and Pylyshyn (pp. ix-xvii). Cambridge, MA: MIT Press.

Kosslyn, S. (1994). Image and brain: The resolution of the imagery debate. Cambridge, MA: MIT Press.

Kosslyn, S., Thompson, W., \& Ganis, G. (2006). The case for mental imagery. Oxford: Oxford University Press.

Liu, J., Harris, A., \& Kanwisher, N. (2002). Stages of processing in face perception: An MEG study. Nature Neuroscience, 5, 910-916.

Nanay, B. (2010). Perception and imagination: A modal perception as mental imagery. Philosophical Studies, 150, 239-254.

Palmieri, T., \& Gauthier, I. (2004). Visual object understanding. Nature Reviews Neuroscience, 5, 291-304.

Perry, J. (2001/2012). Reference and reflexivity, 2nd edn. Stanford, CA: CSLI Publications.

Pylyshyn, Z. (1999). Is vision continuous with cognition? Behavioral and Brain Sciences, 22, 341-365.

Pylyshyn, Z. (2000). Situating vision in the world. Trends in Cognitive Sciences, 4, 197-207.

Pylyshyn, Z. (2001). Visual indexes, preconceptual objects, and situated vision. Cognition, 80, 127-158.

Pylyshyn, Z. (2003). Seeing and visualizing: It's not what you think. Cambridge, MA: MIT Press.

Pylyshyn, Z. (2007). Things and places: How the mind connects with the world. Cambridge, MA: MIT Press.

Pylyshyn, Z. (2009). Perception, representation, and the world: The FINST that binds. In Dedrick \& Trick (Eds.), Computation, cognition, and Pylyshyn (pp. 3-48). Cambridge, MA: MIT Press.

Pylyshyn, Z., \& Storm, R. (1988). Tracking multiple independent targets: Evidence for a parallel tracking mechanism. Spatial Vision, 3, 1-19.

Raftopoulos, A. (2009). Cognition and perception: How do psychology and neural science inform philosophy?. Cambridge, MA: MIT Press.

Raftopoulos, A. (2011). Late vision: Processes and epistemic status. Frontiers in Psychology, 2, 1-12.

Raftopoulos, A., \& Muller, V. C. (2006). Nonconceptual demonstrative reference. Philosophy and Phenomenological Research, 72, 251-285.

Romo, R., \& Salinas, E. (1999). Sensing and deciding in the somatosensory system. Current Opinion in Neurobiology, 9, 487-493.

Schall, J., \& Thompson, K. G. (1999). Neural selection and control of visually guided eye movements. Annual Review of Neuroscience, 22, 241-259.

Shadlen, M., \& Newsome, W. T. (1996). Motion perception: Seeing and deciding. In Proceedings of the national academy of sciences, 93, USA, pp. 628-633.

Strawson, P. (1974). Imagination and perception. In P. Strawson (Ed.), Freedom and resentment (pp. 45-65). London: Methuen.

VanRullen, R., \& Thorpe, S. (2001). The time course of visual processing: From early perception to decision-making. Journal of Cognitive Neuroscience, 13, 454-461.

Warrington, E. (1982). Neuropsychogical studies of object recognition. Philosophical Transactions of the Royal Society of London B: Biological Science, 298, 15-33.

Warrington, E., \& Taylor, A. (1978). Two categorical stages of object perception. Perception, 7, $695-705$. 